\title{
SchreibanfängerInnen im herkunftssprachlichen Russischunterricht
}

\author{
Natalia Brüggemann (Regensburg)
}

\begin{abstract}
Аанная статья посвящена преподаванию русского языка как унаследованного (как семейного, как эритажного) студентам, начинающим осваивать письменный русский язык в немецком университете. В ней обсуждаются конкретные языковые потребности, вытекающие из актуальных научных исследований по многоязычию и русскому как унаследованному в Германии, которые являются основой учебного плана на начальной фазе освоения письменной речи. В статье сформулированы основные принципы преподавания русского языка как унаследованного, такие как осознанное обращение с языком, т.е. способность к языковой рефцексии и анализу языкового материала.
\end{abstract}

\section{1. $\quad$ Einleitung}

Der vorliegende Beitrag beleuchtet Schwerpunkte, die der universitäre herkunftssprachliche Russischunterricht auf der Lernstufe der (Schreib-)AnfängerInnen setzt bzw. setzen sollte. Zu dieser Gruppe gehören diejenigen HerkunftssprecherInnen der zweiten MigrantInnengeneration, deren Sprachkompetenzen auf natürliche (d. h. nicht institutionell gesteuerte), überwiegend mündliche Art erworben wurden und auf Besonderheiten der gesprochenen Sprache und des elterlichen Inputs basieren (Brehmer 2016, Brüggemann 2016). Ihre Sprachkompetenzen sind vergleichbar gut ausgeprägt im Bereich der alltäglichen Sprachkommunikation - v. a. der alltagsbezogenen Sprachrezeption, aber auch der Sprachproduktion. Genauer betrachtet besteht diese Gruppe aus HerkunftssprecherInnen der zweiten Generation, die in Deutschland geboren oder vor der Einschulung immigriert sind und keinen gesteuerten Russischunterricht während der Schulzeit in

Anka Bergmann, Olga Caspers \& Wolfgang Stadler (Hg.)

Didaktik der slawischen Sprachen - Beiträge zum 1. Arbeitskreis in Berlin (12.-14.9.2016)

(C) 2018 innsbruck university press, ISBN 978-3-903187-11-5, DOI 10.15203/3187-11-5 
Deutschland erhalten haben und somit über keine schriftsprachlichen (bildungssprachlichen) Kompetenzen im Russischen verfügen, dafür jedoch im Deutschen (s. auch Ermakova in diesem Sammelband). Sie kommen also zum universitären Russischunterricht als Erwachsene und bringen einerseits eine unvollständige, rein mündlich erworbene Herkunftssprache, andererseits konzeptionell schriftliche Kompetenzen in der Bildungssprache des Deutschen mit, eine Textkompetenz also, auf die sie beim Weitererwerb des Russischen zurückgreifen können.

Es werden hier die einzelnen Bedarfsbereiche in der SchreibanfängerInnenphase thematisiert, die aus der Forschung zur Beschaffenheit der slawischen Herkunftssprachen in den Unterricht einfließen (s. Anstatt 2013, Benmamoun, Montrul \& Polinsky 2013, Bergmann \& Brüggemann i. V., Brehmer \& Mehlhorn 2015, Brüggemann 2016, Kagan \& Rifkin 2000, Polinsky 2015, Polinsky \& Kagan 2007, Polinsky 1997).

\section{HerkunftssprecherInnen der zweiten MigrantInnen- generation im Russischunterricht}

Zunächst soll in Bezug auf die Begriffe „HerkunftssprecherInnen“ und „Herkunftssprache“ (heritage speakers und heritage language) sowie „erste“ und „zweite MigrantInnengeneration“ eine Klärung erfolgen. Ausgangspunkt ist die Definition von heritage speakers und heritage language nach Polinsky \& Kagan (2007: 369f.). Diese definieren HerkunftssprecherInnen des Russischen als Personen, die bis zur Einschulung mit der russischen Sprache aufgewachsen und mehr oder weniger bilingual sind, jedoch ihre Erstsprache aufgrund des Wechsels zu einer anderen dominanten Sprache nicht vollständig erworben haben: „The crucial criterion is that the heritage language was first in the order of acquisition but was not completely acquired because of the individual's switch to another dominant language." (ebd.).

Abweichend davon wird der Begriff „HerkunftssprecherInnen“ bzw. „Herkunftssprache“ ebenfalls bezüglich der sog. „zweiten MigrantInnengeneration“ verwendet. Entscheidend für die Abgrenzung zur „ersten MigrantInnengeneration“ ist das Migrationsalter. Hier werden die Altersgrenzen zwischen „geboren in

Anka Bergmann, Olga Caspers \& Wolfgang Stadler (Hg.)

Didaktik der slawischen Sprachen - Beiträge zum 1. Arbeitskreis in Berlin (12.-14.9.2016)

(C) 2018 innsbruck university press, ISBN 978-3-903187-11-5, DOI 10.15203/3187-11-5 
Deutschland“ und „Migrationsalter bis ca. 10-12 Jahre“ über das Vorschulalter hinaus bis zum Beginn der Pubertät gedehnt (vgl. Anstatt 2012: 4). Die die Zeitspanne abschließende Grenze (ca. 10-12 Jahre) wird auf der einen Seite mit dem Erwerbsbeginn des Deutschen als Zweitsprache angesetzt, auf der anderen Seite mit der Grenze, nach der der natürliche Erstspracherwerb als abgeschlossen und die Sprache als ,perfekt' erworben gilt (zu Erwerbstypen in Bezug auf verschiedene Altersgrenzen bei Erwerbsbeginn und zum möglichen Erwerbsmodus vgl. Klein [2010], Meisel [2007]; umfassende Diskussion über die Begrifflichkeit heritage language und heritage speakers in Benmamoun, Montrul \& Polinsky [2013: 133]).

Im Unterschied zu FremdsprachenlernerInnen, die an Universitäten mit dem Spracherwerb auf Anfängerniveau meist mit Null-Kenntnissen im Russischen beginnen (die FremdsprachenlernerInnen mit institutionell erworbenen Vorkenntnissen steigen meist in späteren Phasen ein), zeigen die HerkunftssprecherInnen zu Beginn des Studiums eine extreme Variabilität hinsichtlich ihrer Sprachkompetenzen und damit spezifische Bedarfsbereiche (vgl. Bergmann \& Brüggemann i. V.). Auf diese hohe Binnen-Heterogenität der Sprachkompetenzen von HerkunftssprecherInnen wurde bereits in der Forschung hingewiesen. Die Sprachkompetenzen der HerkunftssprecherInnen wurden im Sinne eines Kontinuum-Modells erfasst, das sich zwischen zwei Polen erstreckt: vom Pol der rein rezeptiven Kompetenzen (rezeptiver Bilingualismus, basilect heritage) zum Pol, der sich den institutionell gesteuerten Kompetenzen von native speakers annähert (acrolect heritage) (Polinsky 2015: 10, Polinsky \& Kagan 2007: 371f.). Zudem wurden vielfältige sprachinterne, sprachinputbezogene und auch soziolinguistisch motivierte Faktoren genannt, die diese Heterogenität verursachen, wie etwa Migrationsalter und Schulbesuch im Herkunftsland, Ausmaß des gesteuerten Unterrichts im Russischen vor dem Studienbeginn, Qualität und Quantität des elterlichen Inputs in der innerfamiliären Kommunikation, individuelle Sprach- und Lernbegabung, Bildungsgrad der Eltern, Spracheinstellungen und Motivation (für einen Überblick vgl. Anstatt 2011).

Es kommen zu Beginn des Slawistikstudiums HerkunftssprecherInnen in einem Sprachkurs zusammen, die man rezeptive Bilinguale nennen kann, sowie HerkunftssprecherInnen, die bereits eine solide Textkompetenz mitbringen, die also im natürlichen oder auch institutionell gesteuerten Erwerbsmodus ihre Her-

Anka Bergmann, Olga Caspers \& Wolfgang Stadler (Hg.)

Didaktik der slawischen Sprachen - Beiträge zum 1. Arbeitskreis in Berlin (12.-14.9.2016)

(C) 2018 innsbruck university press, ISBN 978-3-903187-11-5, DOI 10.15203/3187-11-5 
kunftssprache erworben haben. Sie alle bringen eine gute Voraussetzung dafür mit, dass sie dem Unterricht in der Zielsprache von Anfang an ohne Probleme folgen können. Dies erlaubt uns, sie innerhalb einer Gruppe zu unterrichten. Es hat wenig Sinn, diese LernerInnen in auf Fremdsprachenstudierende zugeschnittenen AnfängerInnenkursen zu unterrichten, die - bezogen auf das Russische - gerade zu Beginn nicht in der Zielsprache abgehalten werden. Ein derartiger Unterricht würde zu Unterforderung führen und nicht oder nur teilweise auf die Bedürfnisse der LernerInnen eingehen.

Wie o. g., sind die Heterogenität verursachenden Faktoren verschiedenartig und stehen überdies miteinander in Wechselwirkung, sodass die empirische Erforschung ihrer Auswirkung auf die (un-)erreichten Kompetenzen in der Herkunftssprache erschwert ist. Die Auswirkung unterschiedlicher Faktoren ist bisher in der Forschung nicht zufriedenstellend geklärt und bisherige Ergebnisse können nicht generalisiert werden (zur (Un-)Abhängigkeit der Sprachkompetenzen bei HerkunftssprecherInnen von der Qualität oder Quantität des elterlichen Inputs vgl. Brehmer 2016). Keiner der Faktoren führt zwangsläufig zu einer Erklärung, wieso bei den einzelnen HerkunftssprecherInnen ein bestimmtes Niveau der sprachlichen Kompetenzen erreicht oder doch nicht erreicht wird.

Als gesichert gilt, dass die Faktoren „Migrationsalter“ und „Besuch des gesteuerten Unterrichts" die wichtigsten Faktoren darstellen. Denn mit spätem Migrationsalter, z. B. mit neun Jahren, haben die HerkunftssprecherInnen einerseits bereits zwei Jahre schulisches Training in Schriftlichkeit und Orthographie hinter sich, andererseits bis zur Migration keinen eingeschränkten Input in der L1 (also nicht nur das Russisch der Eltern, wie dies bei HerkunftssprecherInnen der Fall ist, die in Deutschland geboren wurden). In einer Untersuchung zur Stabilität der Substantivmorphologie in der Herkunftssprache Russisch (Brüggemann i. V.) konnte gezeigt werden, dass die Faktoren „Migrationsalter“ und „Besuch des gesteuerten Unterrichts“ auf die Beherrschung der grammatischen Substantivkategorien einwirken. In dieser Studie haben drei ProbandInnengruppen (Studierende an der Universität Regensburg, insgesamt 25 ProbandInnen) teilgenommen: HerkunftssprecherInnen mit Migrationsalter vor der Einschulung (0-7 Jahren) und ohne gesteuerten Unterricht, HerkunftssprecherInnen mit Migrationsalter vor der Einschulung (0-7 Jahren) und mit Besuch des gesteuerten Unterrichts,

Anka Bergmann, Olga Caspers \& Wolfgang Stadler (Hg.)

Didaktik der slawischen Sprachen - Beiträge zum 1. Arbeitskreis in Berlin (12.-14.9.2016)

(C) 2018 innsbruck university press, ISBN 978-3-903187-11-5, DOI 10.15203/3187-11-5 
und HerkunftssprecherInnen mit Migrationsalter nach der Einschulung (ab 8-10 Jahren) und ohne gesteuerten Unterricht. Die Untersuchung zeigte des Weiteren, dass die verschiedenen Konstellationen innerhalb der beiden Faktoren „Migrationsalter“ und „Besuch des gesteuerten Unterrichts“ zu verschiedenen Ergebnissen führen. Interessant sind die Ergebnisse bezüglich der letzten Gruppe mit Migrationsalter von acht bis zehn Jahren. Bei ihr sind die Abweichungen vom normativen Kasusgebrauch minimal. Da diese Gruppe noch keinen gesteuerten Unterricht in Deutschland erhalten hat, sondern nur Unterricht in der russischen Grundschule vor der Emigration, sprechen die Daten dafür, dass der Erwerb des Kasussystems mit acht Jahren zum größten Teil als abgeschlossen gelten kann und dass das in der russischen Schule erworbene Kasusystem auch über mehrere Jahre bewahrt wird und nicht oder nicht stark attriiert. Was die Beherrschung der Substantivmorphologie betrifft, unterscheiden sich die HerkunftssprecherInnen dieser Gruppe minimal von den native speakers. Hinsichtlich der Substantivkategorien zeigt die Untersuchung, dass der Faktor „Migrationsalter“ in Zusammenhang mit dem Faktor „,(kein) Besuch des gesteuerten Unterrichts“ für die Binnendifferenzierung im herkunftssprachlichen Unterricht nützlich sein könnte.

\section{Binnendifferenzierung im universitären herkunftssprachlichen Unterricht}

Es versteht sich von selbst, dass die hohe Variabilität der Kompetenzen der HerkunftssprecherInnen besondere Anforderungen für den herkunftssprachlichen Unterricht mit sich bringt, z. B. eine sorgfältige Differenzierung innerhalb der zusammengekommenen Gruppe und eine durchdachte Arbeitsform im Umgang mit Heterogenität.

Diagnostische Verfahren zur Erfassung der sprachlichen Variabilität bei HerkunftssprecherInnen wurden in der Forschung bereits beschrieben. Polinsky (2015) schlägt einen speech rate-Test vor, der die Anzahl der Wörter pro Minute im Output der spontanen Produktion (z. B. bei Bildbeschreibungen) misst. Dabei kann eine Korrelation zwischen dem speech rate-Test und dem Ausmaß des grammatischen Wissens gezeigt werden: "[L] ower proficiency speakers have more

Anka Bergmann, Olga Caspers \& Wolfgang Stadler (Hg.)

Didaktik der slawischen Sprachen - Beiträge zum 1. Arbeitskreis in Berlin (12.-14.9.2016)

(C) 2018 innsbruck university press, ISBN 978-3-903187-11-5, DOI 10.15203/3187-11-5 
difficulty in accessing lexical items, which slows down their speech, but also significantly compromise comprehension and use of structure types that require quick incremental integration of information. In addition, speech rate is connected to utterance planning, and lower proficiency speakers have more problems in that domain as well. Spontaneous speech is thus punctuated by pauses, repetitions, false starts, and codeswitching. As we will show below, knowledge of lexical items and grammatical knowledge are also correlated." (Benmamoun, Montrul \& Polinsky 2013: 142). Ein weiteres diagnostisches Verfahren ist der lexical proficiencyTest. Auch hier konnte eine Korrelation zwischen dem lexikalischen Wissen von heritage speakers und den grammatischen Phänomenen (wie Kongruenz, Kasusmarkierung, Aspekt und Tempusmarkierung) festgestellt werden, und zwar bezogen auf verschiedene Herkunftssprachen (vgl. ebd.).

Daten sowohl zur Flüssigkeit der spontanen Rede als auch zum lexikalischen Wissen können über das Einstufungsgespräch zur Sprachbiographie erhoben werden. Sinnvoll wäre bei diesem Gespräch, das Migrationsalter nach Deutschland zu erfragen, die Dauer des Besuchs der Grundschule im Herkunftsland und des damit verbundenen ersten Trainings in der Interaktion zwischen der gesprochenen und geschriebenen Sprache sowie das Ausmaß des gesteuerten Russischunterrichts während der Schulzeit in Deutschland (falls vorhanden). Allein mit diesen sprachbiographischen Daten kann eine grobe Einteilung in SchreibanfängerInnen und Fortgeschrittene, d. h. HerkunftssprecherInnen mit oder ohne schriftsprachliche Vorkenntnisse erfolgen. Aus der Zusammensetzung der Gruppe - Anteil der SchreibanfängerInnen und Anteil der Fortgeschrittenen - kann schließlich die Arbeitsform und der Umgang mit der Heterogenität abgeleitet werden. Gerade SchreibanfängerInnen benötigen am Anfang des Kurses ein spezielles Programm, das v. a. auf die Verbindung zwischen der gesprochenen und der geschriebenen Sprache und auch auf andere Bedarfsbereiche (s. u.) abzielt. Ob es sinnvoll wäre, die zusammengekommene Gruppe zu teilen oder ein Modell zu praktizieren, gemäß dem am Anfang getrennte Veranstaltungen stattfinden bzw. sich mit gemeinsamen abwechseln, und ob nicht auch zu überlegen wäre, verschiedene Klausuren vorzubereiten, müsste bei der Konzeption des Unterrichts und der Erstellung von Klausurspezifikationen bedacht werden.

Anka Bergmann, Olga Caspers \& Wolfgang Stadler (Hg.)

Didaktik der slawischen Sprachen - Beiträge zum 1. Arbeitskreis in Berlin (12.-14.9.2016)

(C) 2018 innsbruck university press, ISBN 978-3-903187-11-5, DOI 10.15203/3187-11-5 


\section{Bedarfsbereiche bei SchreibanfängerInnen im herkunftssprachlichen Unterricht}

Eine differenzierte Darstellung der wesentlichen Bedarfsbereiche, die im herkunftssprachlichen Unterricht abgedeckt werden müssen, ist in Bergmann \& Brüggemann (i. V.) zu finden. Die folgende tabellarische Übersicht (Tab. 1) zeigt die einzelnen Aspekte auf verschiedenen sprachlichen Beschreibungsebenen mit systemlinguistischem Fokus. Sprachliches Handeln, Pragmatik und normatives Sprechen, interkulturelle Kompetenzen und Landes- und Kulturkunde werden hier nicht berücksichtigt, sind aber ohne Zweifel für den herkunftssprachlichen Unterricht genauso wichtig und müssen entsprechend eingesetzt werden.

Tabelle 1: Bedarfsbereiche im herkunftssprachlichen Unterricht

\begin{tabular}{|l|l|}
\hline Sprachliche Ebene & Bedarfsbereiche im herkunftssprachlichen Unterricht \\
\hline $\begin{array}{l}\text { phonetische und } \\
\text { graphematische } \\
\text { Ebene }\end{array}$ & $\begin{array}{l}\text { Artikulation und Rezeption einzelner Laute; } \\
\text { Sich-Bewusstmachen der Laut-Schrift-Asymmetrie und der } \\
\text { phonetischen Kombinatorik (Reduktion, Assimilation, } \\
\text { Stimmtonverlust); } \\
\text { Sich-Bewusstmachen der Palatalisiertheit und Velarisiertheit } \\
\text { der Konsonanten }\end{array}$ \\
\hline $\begin{array}{l}\text { morphematische } \\
\text { Ebene }\end{array}$ & $\begin{array}{l}\text { Sich-Bewusstmachen der Morphemik; } \\
\text { Verwendung der Morphemik und Wortbildung; } \\
\text { Prinzip der Morphemkonstanz; } \\
\text { Sich-Bewusstmachen der ungebundenen Morphemik }\end{array}$ \\
\hline morphologische & $\begin{array}{l}\text { Stabilisierung der unvollständig erworbenen oder fehlenden } \\
\text { Ebene }\end{array}$ \\
\hline lexikalische Ebene & $\begin{array}{l}\text { Erweiterung des Wortschatzes aus allen funktionalen Stilen; } \\
\text { Lexikalische Kollokationen }\end{array}$ \\
\hline syntaktische Ebene & $\begin{array}{l}\text { Syntaktische Valenzstrukturen } \\
\text { Typen des zusammengesetzten Satzes und Konjunktionen; } \\
\text { Interpunktion }\end{array}$ \\
\hline Textebene & $\begin{array}{l}\text { Erweiterung der Textkompetenz auf verschiedene Textsorten; } \\
\text { Sich-Bewusstmachen der stilistischen Differenziertheit; } \\
\text { Gebrauch adäquater, textkompositionaler sprachlicher Mittel }\end{array}$ \\
\hline
\end{tabular}

Anka Bergmann, Olga Caspers \& Wolfgang Stadler (Hg.)

Didaktik der slawischen Sprachen - Beiträge zum 1. Arbeitskreis in Berlin (12.-14.9.2016)

(C) 2018 innsbruck university press, ISBN 978-3-903187-11-5, DOI 10.15203/3187-11-5 
In diesem Aufsatz möchte ich mich auf den Anfängerunterricht konzentrieren. Für die SchreibanfängerInnen sind insb. Aspekte der phonetischen, graphematischen, morphematischen und morphologischen Beschreibungsebene zentral. Bei den Fortgeschrittenen bestehen die wichtigsten Aufgaben darin, die Ausdrucksfähigkeit der konzeptionellen Schriftlichkeit zu stärken, den Erwerb eines elaborierten Wortschatzes und syntaktischer Komplexität zu fördern, sowie die bildungssprachliche Textkompetenz im Sinne einer Fähigkeit, Texte unterschiedlicher Zwecke und Strukturen selbstständig, sachbezogen und adressatenorientiert zu verfassen, zu trainieren (zur Textkompetenz bei Bilingualen vgl. u. a. Riehl 2014: 121f.).

\subsection{Phonetische und graphematische Beschreibungsebene}

Der herkunftssprachliche Unterricht folgt von Anfang an den Prinzipien, die für die Bewusstmachung des sprachlichen Inventars, seine Funktionen und Strukturen sowie die Regeln ihrer Verbindung und ihrer Verwendung in der geschriebenen und in der gesprochenen Sprache gelten. Im Unterschied zu den Prinzipien des fremdsprachlichen Unterrichts, der primär auf die Herausbildung kommunikativer, interkultureller Fertigkeiten ausgerichtet ist, zielt der herkunftssprachliche Unterricht auf die Herausbildung und Förderung der Sprachbewusstheit ab.

Das Ziel des kognitivierenden herkunftssprachlichen Anfängerunterrichts besteht zunächst darin, die LernerInnen für den Graphembestand zu sensibilisieren. Dabei kommt es besonders auf die Verbindung zwischen Laut und Schrift bzw., weiter gefasst, zwischen der gesprochenen und geschriebenen Sprache an (Phonem-Graphem-Korrespondenz, Gesetze der phonetischen Kombinatorik). Der Fokus liegt des Weiteren auf der Sensibilisierung für das morphematische Inventar und für ausgewählte Orthographieprinzipien (z. B. Morphemkonstanz) sowie für die Vervollständigung von unvollständig erworbenen oder fehlenden morphologischen Kategorien.

Wenn die Phonem-Graphem-Korrespondenzregeln nur für das Deutsche erworben wurden, übertragen die HerkunftssprecherInnen am Anfang des Schreiberwerbs die vorhandenen Regeln des deutschen graphematischen Systems ins Russische. So suchen die SchreibanfängerInnen für lautliche Besonderheiten des

Anka Bergmann, Olga Caspers \& Wolfgang Stadler (Hg.)

Didaktik der slawischen Sprachen - Beiträge zum 1. Arbeitskreis in Berlin (12.-14.9.2016)

(C) 2018 innsbruck university press, ISBN 978-3-903187-11-5, DOI 10.15203/3187-11-5 
Russischen eine Lösung aus dem deutschen graphematischen System. Der folgende Ausschnitt aus meiner Datensammlung (Abb. 1) soll dies illustrieren:

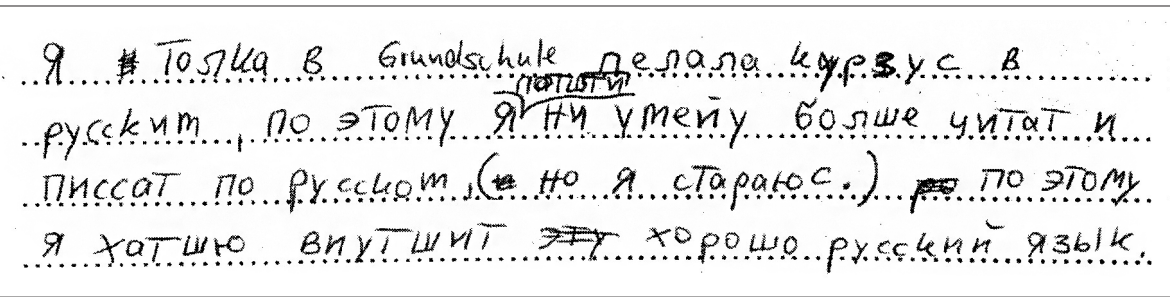

Abbildung 1: Umgang mit lautlichen Besonderheiten (Datensammlung Brüggemann)

(1) Я толка в Grundschule deлала курзус в русскит, по этому я потити ни умейу болше читат и писсат по русскот, (но я стараюос.) по этому я хотшю виутшит хорошо русский язьгк.

,Ich machte nur in der Grundschule einen Russischkurs, deswegen kann ich kaum mehr lesen und schreiben auf Russisch, (aber ich bemühe mich.) deswegen möchte ich gut Russisch erlernen.'

In diesem Beispielsatz sollen die fett markierten Grapheme dieses Transferphänomen auf der graphematischen Ebene veranschaulichen. Für das Graphem $<\mapsto>$ wird die Jotierung ju übernommen, vgl. yмeüy, für das Graphem $<\mathrm{c}>s s$, vgl. nuccam, für $<\mathrm{\varphi}>t s c h$, vgl. nотити, хотию etc. Außerdem kommt es zum materiellen Transfer zwischen den einzelnen Schriftsystemen. Dieser äußert sich z. B. darin, dass innerhalb eines Wortes Graphemmischungen vorkommen, vgl. z. B. das deutsche Graphem $<\mathrm{m}>$ in pycckom. Solche Transfererscheinungen im Alphabet kommen bei bilingualen SprecherInnen auch anderer Lernergruppen (FremdsprachenlernerInnen, MuttersprachlerInnen) durchaus häufig vor. An dieser Stelle soll nur betont werden, dass die Sensibilisierung für den Graphembestand des Russischen und v. a. für die Unterschiede zum Deutschen den Beginn des herkunftssprachlichen Anfängerunterrichts prägt (vgl. auch Beispiele aus Bermel \& Kagan 2000: 420). 
Verschiedene Sprachen unterscheiden sich hinsichtlich der orthographischen (In-)Transparenz zwischen Laut und Schrift. Für ihre Intransparenz sind das Englische und Französische bekannt. Im Slawischen kommt das Bosnisch/Kroatisch/ Serbische dem phonetischen Orthographieprinzip ,1:1-Entsprechung' am nächsten, während das Russische zwar dem Prinzip ebenfalls folgt, aber - wegen der quantitativ-qualitativen Reduktion der unbetonten Vokale und auch wegen der Gesetze der phonetischen Kombinatorik wie Assimilation, Dissimilation, Akkomodation und Stimmtonverlust - eine vorwiegend undurchsichtige PhonemGraphem-Korrespondenz aufweist. So verläuft der Weg zum Orthographieerwerb über die Kenntnisse der Reduktionsprozesse selbst, d. h. über den expliziten Erwerb des phonetischen Wissens im Russischen (z. B. über die Reduktion nach harten Konsonanten, nach weichen und nach harten Zischlauten). Anders wäre es nicht möglich, den SchreibanfängerInnen zu erklären, wieso z. B. маладова nucamesa (Beispiel aus dem Korpus, Fettdruck nur bei Abweichungen aufgrund von Reduktion) nun als молодого писателя, den jungen Schriftsteller' geschrieben wird. Daher wären spezielle Aufgaben zur Phonem-Graphem-Asymmetrie, z. B. in Verbindung mit Reduktionsergebnissen, im herkunftssprachlichen Anfängerunterricht durchaus sinnvoll.

Dieser Weg ,zur Orthographie über die Phonetik' ist ebenfalls hinsichtlich anderer phonetischer Phänomene unvermeidbar. Bermel \& Kagan (2000: 422f.) sprechen von einem weit verbreiteten Verlust der Palatalisierung im ,amerikanischen' Russischen (loss of Russian palatalization) und der übernommenen Jotierung bei der Schreibung der Vokalgrapheme, vgl. das Beispiel Володйa. Diese Beispiele für Abweichungen wegen der fehlenden Palatalisierung der Konsonanten sind ebenfalls für die in Deutschland lebenden HerkunftssprecherInnen typisch und bei den SchreibanfängerInnen weit verbreitet. Wenn man das obere Beispiel (1) noch einmal betrachtet, stellt man fest, dass durchgehend keine Weichheitszeichen verwendet werden. Die fett markierten Grapheme werden also als ,nicht palatalisiert' graphisch abgebildet wie im folgenden Beispiel (2):

(2) Я толка в Grundschule dелала курзус в русккит, по этому я потити ни умейу болше читат и писсат по русскот, (но я старатос.) по этому я хотито виутиит хорошо русский язык.

Anka Bergmann, Olga Caspers \& Wolfgang Stadler (Hg.)

Didaktik der slawischen Sprachen - Beiträge zum 1. Arbeitskreis in Berlin (12.-14.9.2016)

(C) 2018 innsbruck university press, ISBN 978-3-903187-11-5, DOI 10.15203/3187-11-5 
In den orthographischen Regeln der Russischen Akademiegrammatik der Wissenschaften sind Regeln zum Setzen des Weichheitszeichens (auch des Härtezeichens) ausführlich aufgelistet und illustriert. Sie betreffen verschiedene sprachliche Phänomene: neben der o. g. Palatalisiertheit oder Velarisiertheit des Konsonanten z. B. auch das Genus des Substantivs, das Infinitiv-Suffix, die Endung der 2PSg bei Verben, die Regel zur Bildung der Imperativform, die Trennung des weichen Konsonanten und des Vokals durch das j-Graphem u. a. (zur Behandlung der Rechtschreibung im herkunftssprachlichen Unterricht vgl. auch Bermel \& Kagan 2000: 432). Für sinnvoll halte ich jedoch, die Vielfalt der orthographischen Regeln im herkunftssprachlichen Unterricht nicht innerhalb eines separaten Themenblocks aufzulisten, sondern sie portioniert bei inhaltlich passenden Gelegenheiten in den Unterricht einfließen zu lassen: über die phonetisch-graphematische und über die in 4.2 thematisierte morphematische Ebene. Dadurch können die Regeln mit den Ursachen für die Laut-Schrift-Asymmetrie im Russischen verknüpft und die Phonem-Graphem-Korrespondenz transparent gemacht werden (dazu ausführlicher in Bergmann \& Brüggemann i. V.).

\subsection{Morphematische Beschreibungsebene}

Wesentlich für den herkunftssprachlichen Anfängerunterricht ist die Arbeit an der Bewusstmachung des Morpheminventars im Russischen. Die HerkunftssprecherInnen verfügen zwar über das Wissen und die Funktionsweise der Morphemik im Deutschen, z. B. dass dieselben Morpheme in der Schreibung konstant bleiben, können dieses Wissen jedoch nicht direkt auf das Russische übertragen. Die Unkenntnis der lexikalischen und grammatischen Morphematik und die Unfähigkeit, die einzelnen Morpheme zu identifizieren, d. h. zu segmentieren oder auch zu komplexeren Wörtern zu verbinden - seien es gebundene, seien es freie Morpheme, wie Präpositionen -, machen den expliziten Erwerb der russischen Morphemik zu einem weiteren Schwerpunkt im kognitivierenden herkunftssprachlichen Russischunterricht. Eine besondere Stellung erhält in diesem Zusammenhang das Prinzip der Morphem-Konstanz, denn die häufigsten Abweichungen basieren auf der Verletzung dieses Prinzips. Ein und dieselbe Morphemik - Wurzeln oder andere Affixe - kann von SchreibanfängerInnen sogar

Anka Bergmann, Olga Caspers \& Wolfgang Stadler (Hg.)

Didaktik der slawischen Sprachen - Beiträge zum 1. Arbeitskreis in Berlin (12.-14.9.2016)

(C) 2018 innsbruck university press, ISBN 978-3-903187-11-5, DOI 10.15203/3187-11-5 
nebeneinander unterschiedlich geschrieben werden, vgl. die fett markierten Grapheme im Ausschnitt aus dem Korpus:

(3) Wажс поезд прииёл в пать чесов двадиать минут // Сейчас три чиса две минуты

Ihr Zug kam 20 Minuten nach fünf // Jetzt ist es zwei Minuten nach drei

In dieser Textpassage demonstriert der/die VerfasserIn kein Bewusstsein für odnokorennye slova. Ein Vergleich des Wurzel-Morphems yac ,Stunde' mit demselben Morphem unter Betonung findet nicht statt.

Die Morphemik steht in enger Verbindung zur Orthographie. Wenn man die russischen Rechtschreibregeln der Russischen Akademie der Wissenschaften genauer betrachtet, stellt man fest, dass ihre Gliederung nach Paragraphen weitgehend den Typen von Morphemen entspricht (und auch den einzelnen Wortarten). D. h. es sind Regeln, die Wurzelmorpheme, Präfixmorpheme, Suffixmorpheme etc. betreffen. Es wäre also sinnvoll, in die Darstellung der einzelnen Morpheme gleich die entsprechenden orthographischen Regeln einfließen zu lassen, z. B. orthographische Regeln zum Schreiben der Präfixe 3-, c-; Präfixe npe-, npu-; Regeln zum Schreiben der Interfixe -o-, -e-; Postfixe - $c я$ oder - $-c$ etc. (vgl. auch die entsprechenden Übungen in Kagan \& Kudyma 2012: 81, 160ff., 175). Wenn also die Morphemik im herkunftssprachlichen Unterricht behandelt wird, könnten die einzelnen orthographischen Regeln zur Schreibung der Morphemik portioniert eingebunden werden.

\subsection{Morphologische (grammatische) Beschreibungsebene}

Die (In-)Stabilität der morphologischen Kategorien in der Herkunftssprache Russisch wurde im Zusammenhang mit dem sog. unvollständigen Spracherwerb und der sog. Attrition (im Sinne einer sprachlichen Umstrukturierung bei bilingualen Personen) in der Forschung noch nicht tiefgehend behandelt. Einige Untersuchungen zur Beschaffenheit der slawischen Herkunftssprachen (Anstatt 2011, Brüggemann 2016, Brüggemann i. V.) betreffen ausgewählte grammati-

Anka Bergmann, Olga Caspers \& Wolfgang Stadler (Hg.)

Didaktik der slawischen Sprachen - Beiträge zum 1. Arbeitskreis in Berlin (12.-14.9.2016)

(C) 2018 innsbruck university press, ISBN 978-3-903187-11-5, DOI 10.15203/3187-11-5 
sche Kategorien. Die Ergebnisse dieser Studien erlauben uns jedoch, didaktische Konsequenzen zu ziehen und im herkunftssprachlichen Unterricht grammatische Schwerpunkte zu setzen.

Exemplarisch möchte ich auf meine Untersuchung zur (In-)Stabilität der Substantivmorphologie und der Grenzen des unvollständigen Spracherwerbs eingehen (ausführlich in Brüggemann i. V.). An der Untersuchung nahmen insgesamt 25 Studierende der Universität Regensburg teil. Nach ihren sprachbiographischen Daten und der Einteilung gemäß der Faktoren „Migrationsalter“ und „Besuch des gesteuerten Russischunterrichts“ (s. o.) lassen sich die ProbandInnen in drei relativ homogene Gruppen einteilen. Interessant sind v. a. die erhobenen Daten einer dieser drei Gruppen. Zu dieser Gruppe gehören diejenigen HerkunftssprecherInnen $(N=12)$, die in Deutschland geboren wurden oder vor der Grundschule nach Deutschland immigriert sind und noch keinen gesteuerten herkunftssprachlichen Unterricht erhalten haben. Diese Personen wurden untersucht, nachdem sie gerade gelernt hatten, auf Russisch zu lesen und zu schreiben (nach zwei Wochen herkunftssprachlichem Unterricht). Interessant ist diese Gruppe deswegen, weil ihre Herkunftssprache den mündlich erworbenen, vom gesteuerten Unterricht noch unbeeinflussten Zustand widerspiegelt. Es sind also genau diese SchreibanfängerInnen, denen der vorliegende Aufsatz gewidmet ist.

In dieser Studie habe ich den kompletten formal-funktionalen Bestand der Kategorien Kasus-Numerus-Genus, d. h. alle möglichen Kasusformen und alle Kasusfunktionen (Nominativ, Genitiv, Akkusativ, Dativ, Instrumental, Präpositiv) im Zusammenhang mit Genusfunktionen (Maskulinum, Neutrum, Femininum) und Numerusfunktionen (Singular und Plural) unter Berücksichtigung verschiedener Faktoren erfasst und ausgewertet. Diese Faktoren sind: Länge der Endung, Jotierung in der graphischen Realisierung, Betonung und Polyfunktionalität der Endung, Häufigkeit der Kasus-Genus-Numerus-Form im Gebrauch (anhand des Nationalkorpus der russischen Sprache, gesprochene Sprache), Häufigkeit des Lexems und bildungssprachliche Zugehörigkeit des Lexems (im Sinne einer Tendenz). Als Testformat wurde ein Cloze-Test herangezogen, in dem die genannten Faktoren ineinandergreifen. Bestimmte Kasusformen und Funktionen wurden betont/unbetont, bei häufigen/weniger häufigen, bei bildungssprachlichen/nicht bildungssprachlichen Lexemen überprüft. Die Stabilität einer

Anka Bergmann, Olga Caspers \& Wolfgang Stadler (Hg.)

Didaktik der slawischen Sprachen - Beiträge zum 1. Arbeitskreis in Berlin (12.-14.9.2016)

(C) 2018 innsbruck university press, ISBN 978-3-903187-11-5, DOI 10.15203/3187-11-5 
grammatischen Kategorie steht damit an der Schnittstelle zwischen Lexik und Grammatik, Prosodie und kognitiver Privilegiertheit eines Wortes.

Es konnte gezeigt werden, welche einzelnen Subkategorien besonders instabil sind (Dativ, Präpositiv, Instrumental im Plural, mit jotierten Graphemen). Vor allem aber ging aus der Studie hervor, dass (In-)Stabilität ein mehrdimensionales Konstrukt ist, auf das mehrere Faktoren einwirken. Sie ist ein bewegliches Phänomen, d. h. ein und dieselbe Endung wird bei einem häufig in der gesprochenen Sprache vorkommenden Lexem von HerkunftssprecherInnen richtig eingesetzt, bei einem weniger häufigen, schriftsprachlichen Lexem jedoch nicht. Die einzelnen Ergebnisse dieser Untersuchung finden sich in Brüggemann (i. V.).

\section{Vorläufige Schlussfolgerungen}

Die Tatsache, dass (In-)Stabilität ein bewegliches und mehrdimensionales Phänomen ist, begründet den kognitivierenden Lernansatz im herkunftssprachlichen Unterricht und macht die unterschiedliche Herangehensweise im fremdsprachlichen und herkunftssprachlichen Unterricht plausibel. Denn die FremdsprachenlernerInnen besitzen dank der im Unterricht explizit vermittelten Grammatik tendenziell die Fähigkeit, bei jedem unbekannten Wort die richtige Endung zu nennen. Die Kenntnisse der Grammatik sind ihnen meist bewusst (Hartenstein 2014: 191). Die HerkunftssprecherInnen dagegen sind gegenüber der Morphologie ,blind'. Wenn sie ein Wort nicht kennen, können sie keine Formen bilden. Der Formengebrauch ist ihnen meist nicht bewusst und seine Beherrschung zeichnet sich durch eine hohe Unsicherheit aus. Es bleibt also nichts Anderes übrig, als den Stellenwert der Grammatik und des kognitivierenden Lernansatzes im herkunftssprachlichen Unterricht zu erhöhen und neu zu überdenken (dazu s. Bergmann 2015). In dieser Perspektive zeichnen sich viele neue didaktische Forschungsbereiche ab, die noch bearbeitet werden müssen. 


\section{Literaturverzeichnis}

Anstatt, T. (2011). Sprachattrition. Abbau der Erstsprache bei russisch-deutschen Jugendlichen. Wiener Slawistischer Almanach, 67, 7-31.

Anstatt, T. (2012). In zwei Sprachen zu Hause: Slavisch-deutsche Mehrsprachigkeit im Ruhrgebiet. In LWL-Industriemuseum \& D. Osses (Hrsg.), Nach Westen. Zuwanderung aus Osteuropa ins Ruhrgebiet (47-53). Essen: Klartext.

Anstatt, T. (2013). Polnisch als Herkunftssprache: Sprachspezifische grammatische Kategorien bei bilingualen Jugendlichen. In S. Kempgen et al. (Hrsg.), Deutsche Beiträge 15. Internat. Slavistenkongress Minsk 2013 (15-25). München: Otto Sagner.

Benmamoun, E., Montrul, S. \& Polinsky, M. (2013). Heritage Languages and Their Speakers: Opportunities and Challenges for Linguistics. Theoretical Linguistics, 39 (3-4), 129-181.

Bergmann, A. (Hrsg.) (2014). Fachdidaktik Russisch. Eine Einführung. Tübingen.

Bergmann, A. (2015). Fokus auf Sprache im Russischunterricht. Praxis Fremdsprachenunterricht Russisch, 5, 9-11.

Bergmann, A. \& Brüggemann, N. (Hrsg.) (i. V.). Russisch als Herkunftssprache in Deutschland unterrichten. Zu linguistischen Grundlagen und didaktischen Perspektiven des Lehrens und Lernens.

Bermel, N. \& Kagan, O. (2000). The Maintenance of Written Russian in Heritage Speakers. In O. Kagan \& B. Rifkin (Hrsg.), The Learning and Teaching of Slavic Languages and Cultures (405-437). Bloomington, Indiana: Slavica Publishers.

Brehmer, B. \& Mehlhorn, G. (Hrsg.) (2015). Russisch als Herkunftssprache in Deutschland. Ein holistischer Ansatz zur Erforschung des Potenzials von Herkunftssprachen. Zeitschrift für Fremdsprachenforschung, 26 (1), 85-123.

Brehmer, B. (2016). Unveröffentlichter Gastvortrag an der Universität Tübingen „Masse oder Klasse? Zur Bedeutung von Quantität und Qualität des elterlichen Inputs für den Erwerb von Herkunftssprachen (am Beispiel der Herkunftssprache Russisch in Deutschland)“.

Brüggemann, N. (2016). Herkunftssprache Russisch. Unvollständige Grammatik als Folge mündlichen Spracherwerbs. In A. Bazhutkina \& B. Sonnenhauser (Hrsg.), Linguistische Beiträge zur Slavistik. XXII. JungslavistInnen-Treffen in München (37-58). München u. a.: BiblionMedia.

Brüggemann, N. (i. V.). Zur Stabilität der Substantivmorphologie in der Herkunftssprache Russisch. In A. Bergmann \& N. Brüggemann (Hrsg.), Russisch als Herkunftssprache in Deutschland unterrichten. Zu linguistischen Grundlagen und didaktischen Perspektiven des Lehrens und Lernens.

Anka Bergmann, Olga Caspers \& Wolfgang Stadler (Hg.)

Didaktik der slawischen Sprachen - Beiträge zum 1. Arbeitskreis in Berlin (12.-14.9.2016)

(C) 2018 innsbruck university press, ISBN 978-3-903187-11-5, DOI 10.15203/3187-11-5 
Hartenstein, K. (2014). Wortschatz- und Grammatikvermittlung. In A. Bergmann (Hrsg.), Fachdidaktik Russisch. Eine Einführung (190-213). Tübingen: Narr.

Kagan, O. \& Rifkin, B. (Hrsg.) (2000). The Learning and Teaching of Slavic Languages and Cultures. Bloomington, Indiana: Slavica Publishers.

Kagan, O. \& Kudyma, A. (2012). Učimsja pisat’ po-russki. Ekspress-kurs dlja dvujazyčnych vzroslych. Sankt-Peterburg: Zlatoust.

Klein, W. (2010). Typen und Konzepte des Spracherwerbs. In L. Hoffmann (Hrsg.), Sprachwissenschaft. Ein Reader (903-923). Berlin, New York: de Gruyter.

Meisel, J. M. (2007). Mehrsprachigkeit in der frühen Kindheit: Zur Rolle des Alters bei Erwerbsbeginn. In T. Anstatt (Hrsg.), Mehrsprachigkeit bei Kindern und Erwachsenen (93-114). Tübingen: Narr.

Polinsky, M. (1997). American Russian: Language Loss Meets Language Acquisition. In W. Browne et al. (Hrsg.), Annual Workshop on Formal Approaches to Slavic Linguistics. The Cornell Meeting 1995 (370-406). Ann Arbor: Michigan Slavic Publications.

Polinsky, M. \& Kagan, O. (2007). Heritage Languages: In the 'Wild' and in the Classroom. Language and Linguistics Compass, 1 (5), 368-395.

Polinsky, M. (2015). Heritage Languages and Their Speakers: State of The Field, Challenges, Perspectives for Future Work, and Methodologies. Zeitschrift für Fremdsprachenforschung, 26 (1), 7-27.

Riehl, C. M. (2014). Mehrsprachigkeit. Eine Einführung. Darmstadt: WBG. 